\title{
Diseño de un modelo de arquitectura empresarial para publicaciones científicas basado en ADM - TOGAF 9.0
}

\author{
Nemías Saboya Ríos¹, Omar L. Loaiza Jara²a, Danny Lévano Rodríguez ${ }^{3}$ \\ Ingeniería de sistemas, Facultad de Ingeniería y Arquitectura, Universidad Peruana Unión ${ }^{123}$ \\ Orcid ID: https://orcid.org/0000-0002-7166-21971 \\ Orcid ID: https://orcid.org/0000-0002-3262-709X² \\ Orcid ID: https://orcid.org/0000-0002-1783-11053
}

Recibido: 05 de diciembre de 2017

Aceptado: 01 de febrero de 2018

\begin{abstract}
Resumen
El objetivo esta investigación fue proponer el diseño de un modelo de arquitectura empresarial para publicaciones científicas basado en TOGAF 9.0 y modelado en Archimate para la Universidad Peruana Unión La investigación es de tipo tecnológica ya que propone una solución a la necesidad de lograr el posicionamiento a través de un nivel de visibilidad aceptable para publicaciones científicas según líneas de investigación.
\end{abstract}

Palabras clave: arquitectura empresarial; TOGAF; artículos científicos

Design of an enterprise architecture model for scientific publications based on ADM - TOGAF 9.0

\begin{abstract}
Abstrac
The objective of this research study was to design a proposed enterprise architecture model for scientific publications based on TOGAF 9.0 and modeling in Archimate for the Peruvian Union University (Universidad Peruana Unión). This is a technological study since it proposes a solution to the need to achieve positioning through an acceptable level of visibility for scientific publications according to established research fields.
\end{abstract}

Key-words: Enterprise architecture; TOGAF; scientific articles

\section{Introducción}

La educación universitaria en el Perú requiere que los centros universitarios provean profesionales con capacidades necesarias para resolver problemas de diferente magnitud. En este sentido, el Estado Peruano, a través de sus órganos competentes ha generado un marco normativo para que las universidades funcionen bajo parámetros de calidad y logren los fines proyectados en su conjunto. Por otro lado, las tecnologías de información, deben constituirse en soporte para generar iniciativas tecnológicas que hagan que las 
universidades emprendan transformaciones para satisfacer las exigencias del estado $\mathrm{u}$ otros intereses particulares propios de la institución.

Toda organización debe tener presente que el mundo actual está cambiando gracias al empleo de las tecnologías de la información, por ende, una arquitectura de tecnologías de la información sirve para lograr los fines que se persigue. Jaramillo, Cabrera, Abad, y Torres. (2016), mencionan que el ambiente de las organizaciones cambia conforme se desarrollan innovaciones tecnológicas, donde surgen oportunidades que deben ser aprovechadas.

Para Arango, Londoño \& Zapata (2010), la Arquitectura empresarial en una organización es una representación integral, lo que conlleva a que se establezca una visión clara con objetivos y metas congruentes al negocio de la empresa. El alineamiento inicia desde la gestión estratégica considerando la misión, visión, lineamientos e indicadores estratégicos, hasta a una descripción técnica que se le conoce como construcción de la arquitectura, integrada, que detalla sistematizadamente la estructura actual y futura de los procesos de la organización; estos a su vez como pieza fundamental necesita la incorporación de elementos sustanciales para que funcione de manera óptima como los procesos donde se evidencia modelos de negocio, la estructura organizacional que representa a personas y estructuras administrativa, y finalmente a las tecnologías de información que radica en la incorporación de elementos informáticos como: aplicaciones, repositorios de información, esquemas de infraestructura tecnológica y seguridad informática.

El propósito de esta investigación es proponer un modelo de arquitectura empresarial para publicaciones científicas basado en el marco de trabajo TOGAF 9.0 y utilizando el lenguaje de modelado Archimate 3.1 en la Universidad Peruana Unión (UPeU) enfocado al eje de investigación, sección de publicación de libros y artículos, en ese sentido, se consideró los procesos e indicadores que establece el plan estratégico. Este estudio inicia describiendo el contexto en el ambiente externo a la UPeU, tomando en cuenta las normas de las entidades estatales en materia de acreditación y licenciamiento en la educación superior universitaria, el direccionamiento estratégico (misión, visión, objetivos estratégicos e indicadores en materia de publicaciones científicas).

La descripción anterior debe permitir tener información suficiente para formular concerns, que permitan elaborar una vista motivacional de arquitectura empresarial, esto a su vez otorga un modelo sustraído del contexto organizacional alineados al plan estratégico actual para ser convertidos en objetivos y metas del modelo de arquitectura empresarial. Adicionalmente son diseñados los procesos (AS-IS) organizacionales de publicaciones, tal cual se ejecutan en la realidad objeto de estudio, así como los procesos en versión To be que serían los procesos mejorados y propuestos para el estudio. 


\subsection{Arquitectura empresarial}

\subsubsection{Origen de la arquitectura empresarial (EA)}

La arquitectura empresarial tiene sus principios desde 1987, cuando Zachman (1987) publica en el diario IBM System un artículo titulado "Un marco para la arquitectura de sistemas de información”. En ese artículo. Zachman establece un fuerte desafío a las organizaciones, indicando que lo único que permitirá guiar a las organizaciones en los próximos años hasta nuestros días, será el tener un enfoque a la automatización de la organización.

El enfoque proporcionado por Zachman fue de mucha influencia en los estados unidos y es así como el Departamento de Defensa (DoD), crea una arquitectura empresarial denomina Technical Architecture framwork for information Manager (TAFIM), dicha Modelo de arquitectura empresarial proporcionó orientación a nivel de empresa para la evolución de la infraestructura técnica del DoD. Identifica los servicios, las normas, los conceptos, los componentes y las configuraciones que pueden utilizarse para guiar el desarrollo de arquitecturas técnicas que satisfagan requisitos específicos de la misión.

Proceso de planificación de la arquitectura basada en estándares (SBA). Definido por el TAFIM, consta de siete fases distintas, así como se muestra en la figura 1, pero interdependientes. Cada fase del proceso SBA tiene la intención de crear productos y / o documentos específicos que guíen la fase subsiguiente. Las siete fases se describen brevemente a continuación.

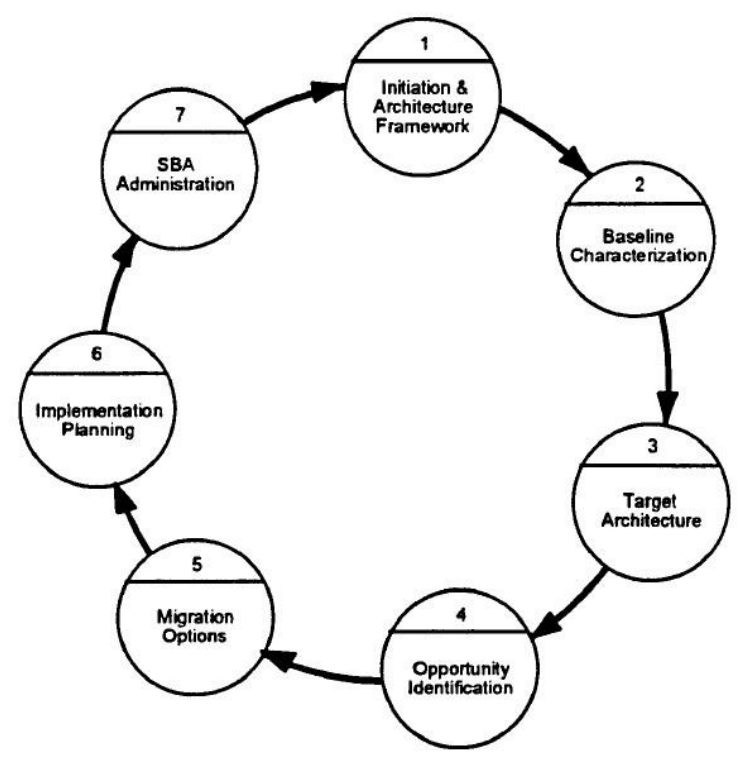

Figura 1. Modelo TAFIM de The Open Group Architecture Framework, recuperado de: https://wikivisually.com/wiki/The_Open_Group_Architecture_Framework

Fase 1, Marco de Iniciación y Arquitectura: La metodología comienza con una adecuada iniciación del proceso dentro de la organización. Esto implica desarrollar un conjunto de impulsores estratégicos para la 
organización. Además, se revisa o se construye un modelo de negocio para establecer un modelo de operación de objetivos estratégicos.

Fase 2. Caracterización de línea de base: Esta fase de puesta a tierra pretende determinar la arquitectura actual de la organización. Es una evaluación del entorno actual, que da lugar a una caracterización en cuatro dimensiones o puntos de vista clave: el trabajo, la información, la aplicación y la tecnología.

Fase 3. Arquitectura meta: Las diversas visiones del marco se modelan en términos de una arquitectura deseable de la blanco, generalmente 3 a 5 años en el futuro.

Fase 4. Identificación de Oportunidades: Pasar de la reflexión conceptual a la realidad práctica y la implementación, con la determinación de los proyectos de desarrollo necesarios.

Fase 5. Opciones de migración: Vincula la realidad del presente con la conveniencia de la arquitectura objetivo estableciendo una o más mesetas que representen etapas prácticas de migración.

Fase 6. Planificación de la implementación: La fase da como resultado un plan de implementación detallado para la primera meseta del esfuerzo migratorio.

Fase 7. Institucionalización del Proceso ITA: Esta fase pretende mantener la arquitectura viva y bien mejorando continuamente.

\subsection{2. ¿Qué es la arquitectura empresarial?}

Niemi E \& Pekkola S (2016), lo definen como la competencia de la organización que "incluye todos los modelos necesarios para administrar y desarrollar una organización y tiene una visión holística de sus procesos de negocio, sistemas de información e infraestructura tecnológica.” Asimismo Mejía (2013), indica que la arquitectura empresarial es un método que alinea a la organización en todos sus niveles, dándole una visión integral de los objetivos estratégicos, permitiéndole de esta manera una alineación de procesos, información, aplicaciones y toda la infraestructura tecnológica de la organización.

Por otro lado, Lankhorst (2005), declara también que "La arquitectura empresarial es un conjunto coherente de principios, métodos y modelos que se utilizan en el diseño y la realización a nivel empresarial de la estructura organizacional, los procesos de negocio, los sistemas de información y la infraestructura"

\subsubsection{La arquitectura empresarial y sus beneficios}

Arango, Londoño \& Zapata (2010) presentan un conjunto de beneficios que proporciona la arquitectura empresarial, estos beneficios generados por la visión holística que provee su modelo de gestión.

1. La Arquitectura empresarial permite que la organización se ubique en su contexto y se articula bajo una estructura coherente, en todos sus niveles. 
2. La Arquitectura empresarial integra los niveles de la organización desde los aspectos de planificación, operaciones del negocio y aspectos tecnológicos arrutándolos bajo los mismos objetivos.

4. La Arquitectura empresarial elimina el desconocimiento entre los procesos del negocio y la tecnología que la soporta y la unifica llevándola a lo exigido y requerido por la dirección estratégica.

5. La Arquitectura empresarial unifica, optimiza y/o elimina procesos y tecnologías que generan costos operacionales, llevándolos a la mejora continua y con sentido.

\subsubsection{TOGAF y La arquitectura empresarial}

TOGAF es un marco de trabajo propuesto por el OpenGroup que está basado en los principios de la arquitectura empresarial. Es un modelo iterativo que establece un conjunto de buenas prácticas para llevar a la organización a una alineación tecnológica de los procesos y objetivos estratégicos. La propuesta metodológica de TOGAFestá centralizada en el Método de desarrollo de arquitectura (ADM), este presenta un ciclo de fases que permite definir un modelo de negocio alineando los objetivos, estratégicas. Procesos y tecnología de la organización (ver figura 2).

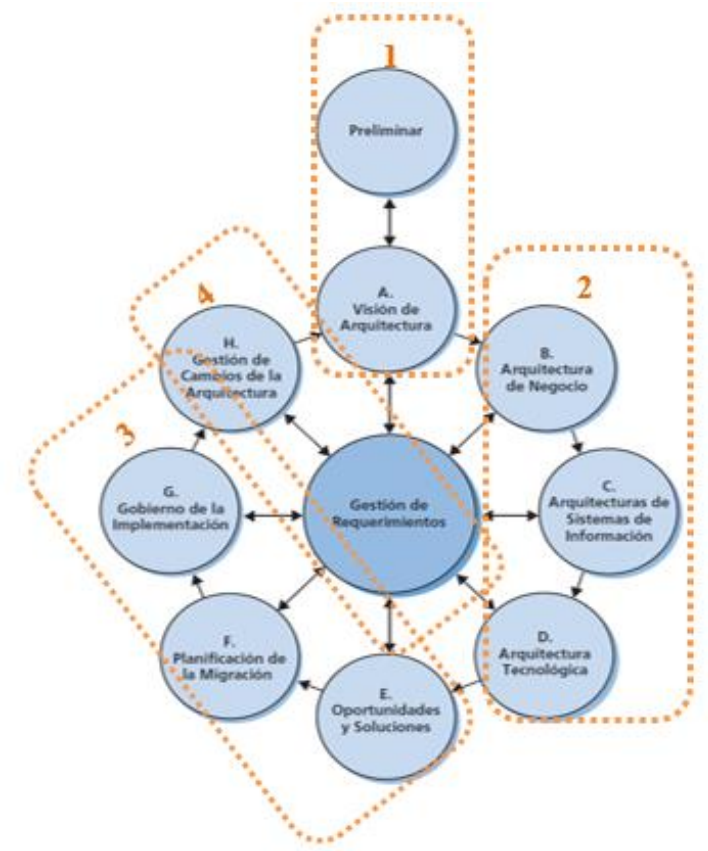

1. Identificar la estrategia de la organización y obtener su compromiso y participación

2. Identificar (entender) la situación actual y diseñar (proponer) la situación futura en relación a los dominios de la arquitectura de negocio, sistemas de información y tecnología.

3. Implementación de la transformación

4. Monitoreo y gestión de la arquitectura para que se mantenga alineada a los requerimientos del negocio.

Figura 2. Estructura ADM-TOGAF, por Ares (2013) recuperado de: https://es.slideshare.net/netmindit/introduccin-a-togafpara-el-desarrollo-de-enterprise-architecture.

La fase preliminar define la orientación de la organización, en esta fase se define y examina el contexto de la organización y se identifican la estructura organizacional.

En la fase A se desarrolla una actividad principal del modelo, se establecen los alcances, limitaciones, restricciones del futuro modelo de arquitectura empresarial. En esta etapa se presenta y se crea la visión de 
la Arquitectura, se identifican y describen a los interesados, se valida la orientación del negocio y se crea en una vista de alto nivel lo que será el trabajo de arquitectura.

La fase B, C y D es donde se estructura y define la arquitectura en cuatro dominios de la arquitectura; Negocio, Sistemas de información - aplicaciones, Sistemas de información - datos y tecnología.

La Fase E, presenta criterios para esquematizar una planificación futura de la implementación de la arquitectura, una de las tareas primordiales es la elaboración del Roadmap, el cual presenta la serie de jugadas a realizar para implementar la tecnología en la organización.

La Fase F, presenta un plan detallado de la implantación y migración de la arquitectura.

La Fase G, establece una estrategia de supervisión y monitoreo de la implementación, define y publica acuerdos o contratos formales para la implementación de la arquitectura.

La Fase H, provee un plan de gestión de cambios para aseguras que la arquitectura establecida en la organización responda de manera continua a las necesidades de ella, para que de esta manera se maximice el valor de la arquitectura para el negocio.

\subsubsection{Lenguaje de modelados (Archimate)}

Archimate es en términos del Open Group un lenguaje de modelado que permite representar gráficamente las capas de una arquitectura empresarial, de modo tal, que los arquitectos empresariales puedan abstraer elementos relevantes tras una prognosis de su ambiente de negocios, $\mathrm{y}$, transitar fácilmente a modelos tecnológicos; en un sentido práctico, se representa gráficamente como se quiere construir una arquitectura empresarial con TI que responda a las necesidades estratégicas del negocio.

Está compuesto de un conjunto de estereotipos con un significado claro y definido de su representación y especificación. Varios símbolos utilizados de manera conjunta permiten modelar una vista arquitectónica, de acuerdo al interés del Arquitecto al recorrer la metodología ADM. Así, se tienen estereotipos para representar actores, drivers, metas, restricciones para la vista motivacional; también se tienen elemento de dibujo para modelar artefactos de la capa de negocios como: procesos, actividades, objetos de negocio y otros; en cuanto a la capa de aplicaciones se puede dibujar el uso de aplicaciones, servicios, interfaces, que representen el propósito de arquitecturas de software, las piezas principales para su funcionamiento desde un punto de vista lógico; y por último, estereotipos para simbolizar artefactos físicos de hardware. Todos estos símbolos deberán permitir representar la estructura global a lograr para que las TI se alineen a la estrategia de negocios corporativa. 


\subsubsection{Procesos de publicaciones científicas}

La producción científica de cualquier índole pasa por un proceso editorial riguroso y denso de actividades hasta ser publicado en una revista científica. Este proceso involucra la participación de diferentes actores, entre ellos tenemos: escritores, editores de revistas, editoriales y bibliotecas digitales. Un proceso riguroso garantiza que cualquier publicación sea enriquecedora y aporte conocimientos y experiencia incremental a una ciencia en particular o general.

Previo al proceso de publicación, generalmente los autores suelen seleccionar a qué revista científica deberían publicar, aquí un conjunto de criterios acompañan la selección como por ejemplo: factor de impacto de la revista (ISI, JCR, etc), alcance temático, periodicidad, idioma, etc. Efectuada esta actividad puede iniciarse el proceso de publicación.

Los procesos de publicación comienzan cuando los cuerpos editoriales de cualquier revista convoca a la comunidad científica a publicar sus manuscritos, o, los autores se ponen en contacto; para cualquier de las formas, la forma actual de hacerlo es usando correos electrónicos o carga de archivos desde una página web.

Una vez recibido el manuscrito, generalmente pasa por un filtro inicial que permitirá saber si el manuscrito cumple con el estilo de redacción, si está dentro del alcance temático, respeta cuestiones éticas y está acompañado de la documentación que una revista exija, de ser así, es admitido a sumisión. Luego, es sometido a una revisión, tanto de la forma como del fondo del documento; en este punto, los revisores pueden conocer o no a los autores de los manuscritos, dependiendo de las políticas de trabajo de la revista científica en cuestión; en cuanto al fondo del manuscrito, suelen usarse tecnologías anti plagio para detectar copias indebidas de textos; se revisan la coherencia entre las partes del documento, se analiza la sección metodológica para saber si cumple con los principios de reproducibilidad y falsación. De haber observaciones, estas deben ser subsanadas en un plazo razonable, y el levantamiento de observaciones debidamente sustentadas. Cuando el comité revisor no tiene más observaciones, recomienda su publicación para para pasar al proceso editorial de diseño gráfico y formato físico en la revista.

El citación anterior de actividades no señala necesariamente un orden pre establecido o prescrito, sino que es una factorización genérica, que por lo general sucede; cada revista se reserva el derecho ejecutar sus actividades con arreglo a sus principios y fines. Queda por si sentada, la idea que existe un proceso, accionado por actores y soportado por herramientas tecnológicas que gestionen las actividades y la información que se genere. 


\section{Materiales y métodos}

\subsection{Participantes}

Para este estudio se consideró como caso a la UPeU y los participantes fueron docentes y administradores relacionados con la investigación científica, quienes participaron como evaluadores y validadores del modelo propuesto.

\subsection{Instrumentos}

La información se colectó a través de una guía de entrevista semi estructurada, que fueron aplicadas a interlocutores válidos respecto del proceso de publicación científica; en dicho instrumento se formularon ítems para comprender elementos de la gestión estratégica como misión, visión.

También se utilizaron fichas para el acopio de información mediante la técnica de revisión documental. Los documentos que se revisaron fueron documentos de gestión como Plan estratégico, Plan operativo anual (POA), Reglamento general de investigación, estándares de calidad del SINEACE.

Por otro lado, para validar el modelo, se utilizó la técnica de revisión documental y se elaboró un instrumento de observación que fueron validados por expertos, que sirvió para la contratación y validación del modelo de arquitectura empresarial.

\section{Resultados y Discusión}

\subsection{Fase preliminar}

En esta fase se realizó un análisis general de la Universidad Peruana Unión (UPeU), institución educativa confesional de una denominación religiosa en el Perú; cuenta con una sede principal en la ciudad de lima y dos fíales (Juliaca y Tarapoto) y de la visión y misión de la institución. Para cumplir con su direccionamiento estratégico, la institución articula estratégicamente ejes estratégicos que se desprenden de la visión estableciendo objetivos institucionales que apoyan a las gestiones de la calidad universitaria de la institución y ante los requerimientos de calidad educativa propuestas por el estado y la Iglesia Adventista del Séptimo Día [IASD]

El eje de investigación de la UPeU actualmente precisa del desarrollo y logro de: proyectos de investigación e innovación, elaboración tesis, publicaciones científicas y participación en eventos científicos, estos elementos se encuentran contemplados en el POA y están soportados por un conjunto de procesos formalizados (Mapa de procesos) que ayudan al cumplimiento de dichos elementos. En este estudio se enfocó el elemento de publicaciones científicas y los procesos que lo soportan. 


\subsection{Fase A: Visión de la arquitectura}

En esta fase se identificaron concerns; se tomó en cuenta el statu quo del contexto de la UPeU en materia de investigación universitaria en el Perú, así como de entrevistas efectuadas a stakeholders tales como el director general de investigación, director de escuelas profesionales, docentes investigadores y estudiantes, los resultados ayudó a identificar la preocupación más relevante que fluyen en el cumplimiento de la visión institucional.

El concern más relevante para el estudio fue "Lograr posicionamiento a través de un nivel de visibilidad aceptable por publicaciones científicas según líneas de investigación”, en estudiantes y docentes. Además, se identificó una lista inicial de potenciales drivers tecnológicos que pueden coadyuvar a facilitar el cumplimiento de la visión en relación al concern tratado en el estudio, insumo que fue utilizado para elaborar el modelo de arquitectura empresarial (ver tabla 1).

Tabla 1. Matriz de relación de concern, con drives tecnológicos

\begin{tabular}{|c|c|c|c|c|}
\hline No. & $\begin{array}{c}\text { Concern } \\
\text { general }\end{array}$ & Concern específicos & Tipo & $\begin{array}{c}\text { Drivers } \\
\text { Tecnológicos }\end{array}$ \\
\hline 1 & $\begin{array}{l}\text { Lograr posiciona- miento a } \\
\text { través de un nivel de } \\
\text { visibilidad aceptable por } \\
\text { publicaciones científicas } \\
\text { según líneas de } \\
\text { investigación. }\end{array}$ & $\begin{array}{l}\text { 1. Lograr posiciona- miento a través de } \\
\text { un nivel de visibilidad aceptable por } \\
\text { publicaciones científicas en bases de } \\
\text { datos de alto impacto según líneas de } \\
\text { investigación institucionales, a nivel } \\
\text { de estudiantes. } \\
\text { 2. Lograr posiciona- miento a través de } \\
\text { un nivel de visibilidad aceptable por } \\
\text { publicaciones científicas según líneas } \\
\text { de investigación institucionales, a } \\
\text { nivel de docentes. }\end{array}$ & $\mathrm{N}$ & $\begin{array}{l}\text { 1. Software } \\
\text { colaborativo: } \\
\text { PlagScan, OCS, } \\
\text { Mendeley u otros. } \\
\text { 2. Redes sociales } \\
\text { 3. Análisis } \\
\text { predictivo } \\
\text { 4. Web semántica. }\end{array}$ \\
\hline
\end{tabular}

$\mathrm{O}=$ Oportunidad $\mathrm{P}=$ Problema $\quad \mathrm{N}=$ Necesidad $\quad \mathrm{X}=$ Sin identificar, pero es importante

La figura 3, permite visualizar el diagrama de vista motivacional derivada de la necesidad de generar un modelo de arquitectura empresarial relacionado al tema de publicaciones científica en la UPeU. Asimismo se identifican a los stakeholdres que están directamente relacionados al caso: funcionarios académicos y administrativos, docentes y estudiantes, estos stakeholdres son los encargados de posicionar a la universidad por medio de sus publicaciones de los resultados de las investigaciones que realizan.

La UPeU como estrategia realizan actividades anuales evidenciadas en su Plan operativo anual para el cumplimiento de metas y estos a su vez está relacionada a indicadores según ejes; en el caso de investigación; según necesidad del estudio se consideró dos metas: uno relacionado a docente: "más del 30\% de docentes a tiempo completo publican en revistas de alto impacto" y el otro a estudiantes: "más de los $20 \%$ matriculados publican sus trabajos de investigación”.

La meta en relación a docentes, está relacionada y dependerá de los resultados del porcentaje de artículos aceptados en revistas de alto impacto, el número de libros y/o capítulos de libros científicos financiados por 
la UPeU o autofinanciados por Autores (comunidad universitaria). Estas metas pueden ser afectadas por los resultados siguientes: De manera negativa si los artículos, libros o capítulos de libros son rechazados u observados; y de manera positiva cuando estos logran se aceptados para su consecuente publicación. Para la meta en relación a estudiantes está relacionada con los resultados de las publicaciones de sus artículos científicos empíricos y con los trabajos de investigación en repositorios.

Para garantizar el éxito de las metas y la propuesta se necesita contar con capacidades en: a) gestión de publicaciones de libros o capítulos de libros y b) gestión de artículos científicos en revistas. Estas capacidades deben ser soportadas por recursos (personas y procesos) los cuales se detallan en la figura 3

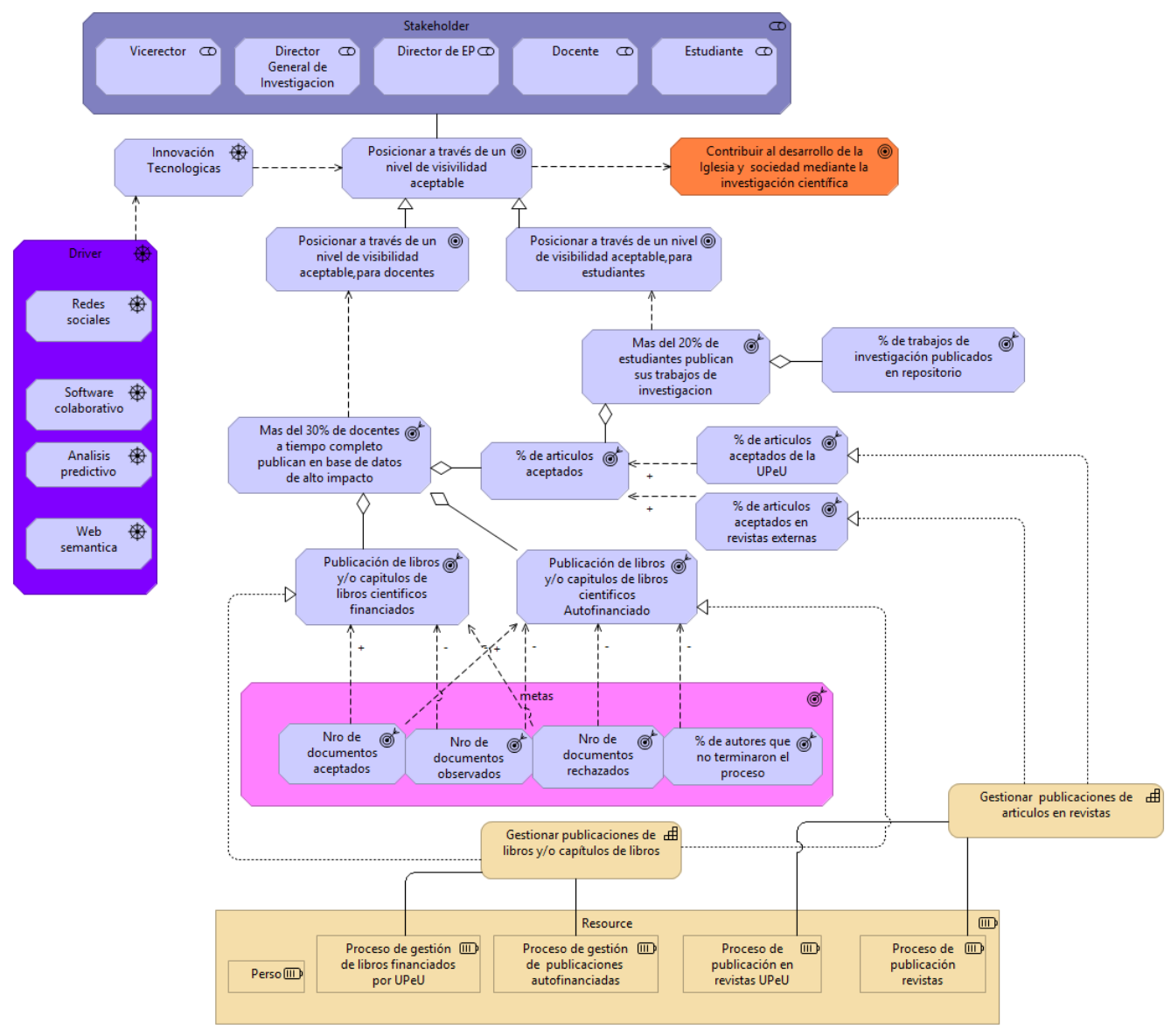

Figura 3. Vista motivacional de publicaciones científicas

\subsection{Fase B: Arquitectura de negocio}

En esta fase se elaboraron los diagramas de procesos AS-IS y TO-BE que dan la capacidad de lograr la meta establecida en relación a los objetivos organizacionales; para el estudio se consideró los siguientes procesos: proceso de publicación de artículos en revistas de la UPeU, proceso de publicación de artículos en revistas externas, proceso de publicación de libros y financiados por la UPeU, proceso de publicación de libros autofinanciados, Para efectos de este articulo solo ser presentará el proceso de publicaciones en revistas externas. 


\subsubsection{Publicación de artículos en revistas externas}

\section{AS-IS}

La figura 4 presenta el conjunto de actividades que desarrolla el proceso de publicación de artículos en revistas externas; como se puede observar el proceso permite la interacción entre el actor del negocio, el Autor, y el rol de negocio, comité editorial; dicha interacción se desarrolla a través de un conjunto de actividades desde la actividad, someter artículo y pasando por actividades que permiten generar entregables que darán persistencia a la información dentro del proceso, hasta llegar a la actividad, publicar artículo, logrando tener de esta manera tener como elemento principal el artículo publicado en revistas de la UPeU.

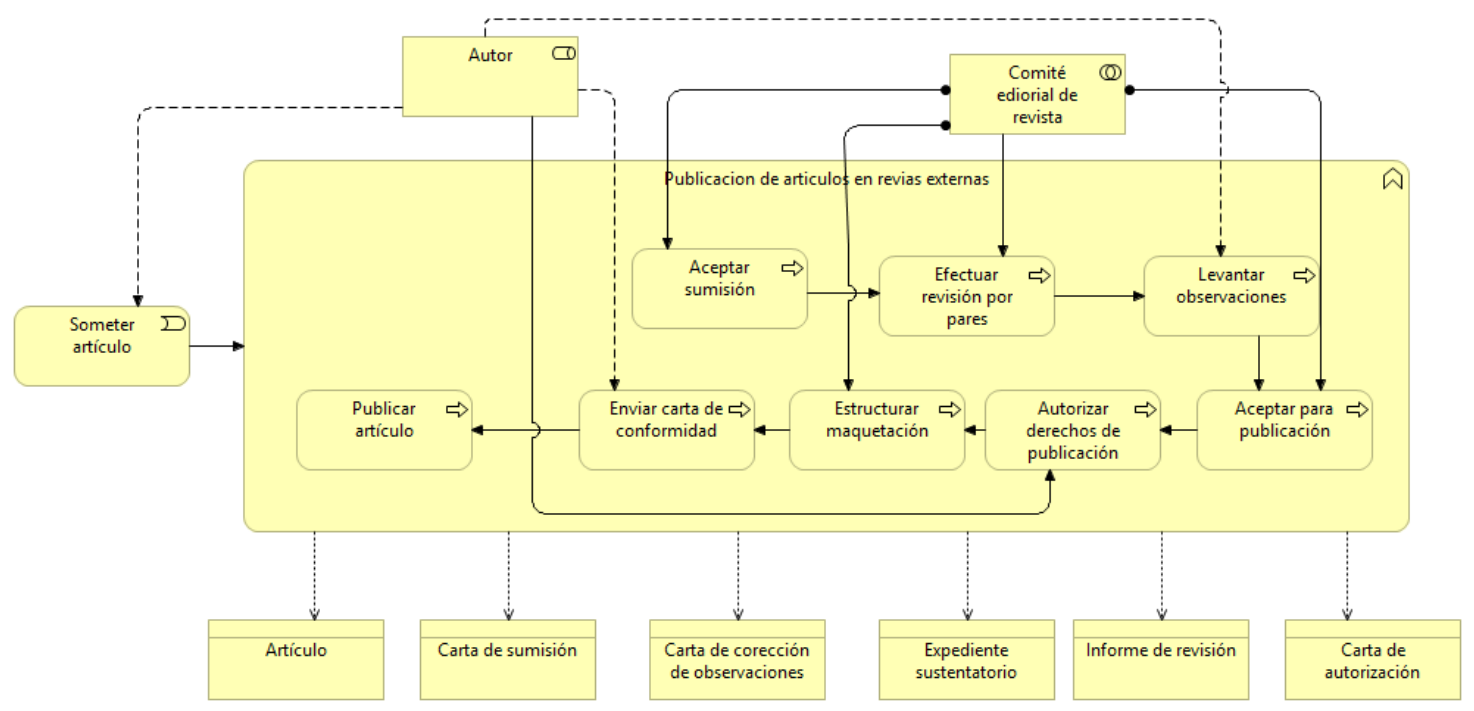

Figura 4. Modelo actual de publicación en revistas externas

\section{TO-BE}

Para el mismo proceso de publicar artículos en revistas externas, la figura 5 propone realizar un ajuste de las actividades con la finalidad de orientar el proceso a ver al autor como un cliente en vez de verlo como un trabajador del proceso y asimismo esto permitirá tener una visión orientada a llevar el proceso a la automatización, así como lo presenta el modelo BPMN. 


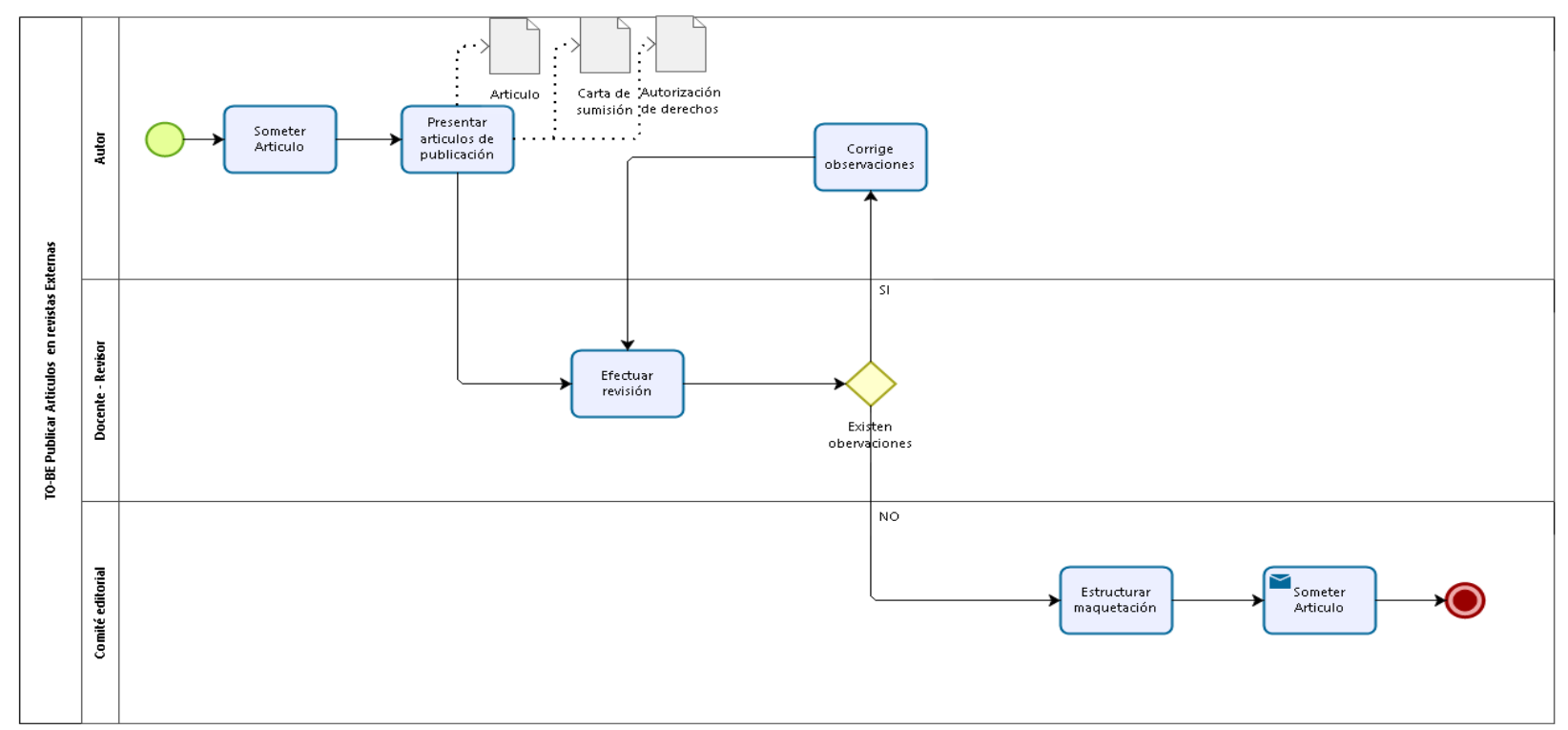

Figura 5. Modelo propuesto de publicación en revistas externas con BPMN con fines de automatización

De manera complementaria un modelo de arquitectura empresarial desarrolla el gap análisis (ver figura 6), dentro de esta tarea, se identifican que actividades serán reemplazadas, adicionadas o suprimidas del proceso AS-IS en contraste al proceso TO BE, esto permite presentar y sustentar de forma técnica y resumida los cambios generados de un proceso a otro, justificando el cambio

GAP ANALYSIS: AS IS - TO BE

\begin{tabular}{|c|c|c|c|c|c|c|c|c|c|c|}
\hline Baseline $\rightarrow$ & & & & & & Autorizar & & & & \\
\hline Target & $\begin{array}{l}\text { Someter } \\
\text { articulo }\end{array}$ & $\begin{array}{l}\text { para } \\
\text { sumisión }\end{array}$ & $\begin{array}{l}\text { revisión por } \\
\text { pares }\end{array}$ & $\begin{array}{c}\text { Levantar } \\
\text { observaciones }\end{array}$ & $\begin{array}{l}\text { para } \\
\text { publicación }\end{array}$ & $\begin{array}{l}\text { derechos } \\
\text { de } \\
\text { publicación }\end{array}$ & $\begin{array}{l}\text { Estructurar } \\
\text { maquetación }\end{array}$ & $\begin{array}{c}\text { de } \\
\text { conformidad }\end{array}$ & $\begin{array}{l}\text { Publicar } \\
\text { articulo }\end{array}$ & Nuevos \\
\hline $\begin{array}{l}\text { Someter } \\
\text { Articulo }\end{array}$ & & & & & & & & & & \\
\hline $\begin{array}{l}\text { Seleccionar } \\
\text { revista de } \mathrm{BD}\end{array}$ & & $\begin{array}{l}\text { Se coloca } \\
\text { una } \\
\text { Aplicación }\end{array}$ & & & & & & & & \\
\hline $\begin{array}{l}\text { Presentar } \\
\text { requisitos de } \\
\text { publicación }\end{array}$ & & & & & & & & & & $\begin{array}{l}\text { Presentar } \\
\text { requisitos } \\
\text { de } \\
\text { publicación }\end{array}$ \\
\hline $\begin{array}{l}\text { Efectuar } \\
\text { revisión }\end{array}$ & & & $\begin{array}{l}\text { Modificado } \\
\text { (Automatizado } \\
\text { ML) }\end{array}$ & & & & & & & \\
\hline $\begin{array}{l}\text { Levantar } \\
\text { observaciones }\end{array}$ & & & & & & & & & & \\
\hline $\begin{array}{l}\text { Aceptar } \\
\text { publicación }\end{array}$ & & & & & & & & & & \\
\hline $\begin{array}{l}\text { Estructurar } \\
\text { maquetación }\end{array}$ & & & & & & & & & & \\
\hline $\begin{array}{l}\text { Someter } \\
\text { articulos a } \\
\text { revistas } \\
\text { externas }\end{array}$ & & & & & & & & & & \\
\hline Eliminados & & $\begin{array}{c}\text { Someter } \\
\text { para } \\
\text { publicación }\end{array}$ & & & & $\begin{array}{c}\text { Autorizar } \\
\text { derechos } \\
\text { de } \\
\text { publicación }\end{array}$ & & $\begin{array}{c}\text { Enviar carta } \\
\text { de } \\
\text { conformidad }\end{array}$ & & \\
\hline
\end{tabular}

Figura 6. Análisis gap para lograr el proceso To Be en publicación en revistas externas

El gap análisis de la figura 6 expresa las acciones que necesitan efectuar en un roadmap, para llegar al proceso en su versión TO BE desde la situación actual AS IS. Por ejemplo, se coloca una aplicación para 
gestionar la sumisión de sumisiones, otra para para automatizar la revisión de manuscritos mediante aplicaciones dotados de algoritmos de machine learning,

\subsection{Fase C y D: Arquitectura de Servicios y Tecnológica}

\subsubsection{Publicación de artículos en revistas- Actividad efectuar revisión}

En esta fase se desarrolló la arquitectura de la capa de aplicaciones, datos y tecnológica; como se puede ver la actividad efectuar revisión del proceso de publicación de artículos requerirá 2 servicios de aplicación que son Realizar revisión externa y realizar revisión interna estas 2 se encontrarán soportadas por una función de aplicación llamada Gestionar revisiones el cual requiere de los objetos artículo, contenido y revisiones, los cuales permitirán dar persistencia a los datos procesados en la función de aplicación, el cual interactúa de manera externa a través de un componente de publicaciones el cual es presentado a través de un acceso web y un acceso móvil; finalmente la tecnología requerida será un Enterprise Service Bus (ESB) el cual funcionará como middleware entre los servidores oracles HP, Redhat y un servidor Backup (ver figura 7)

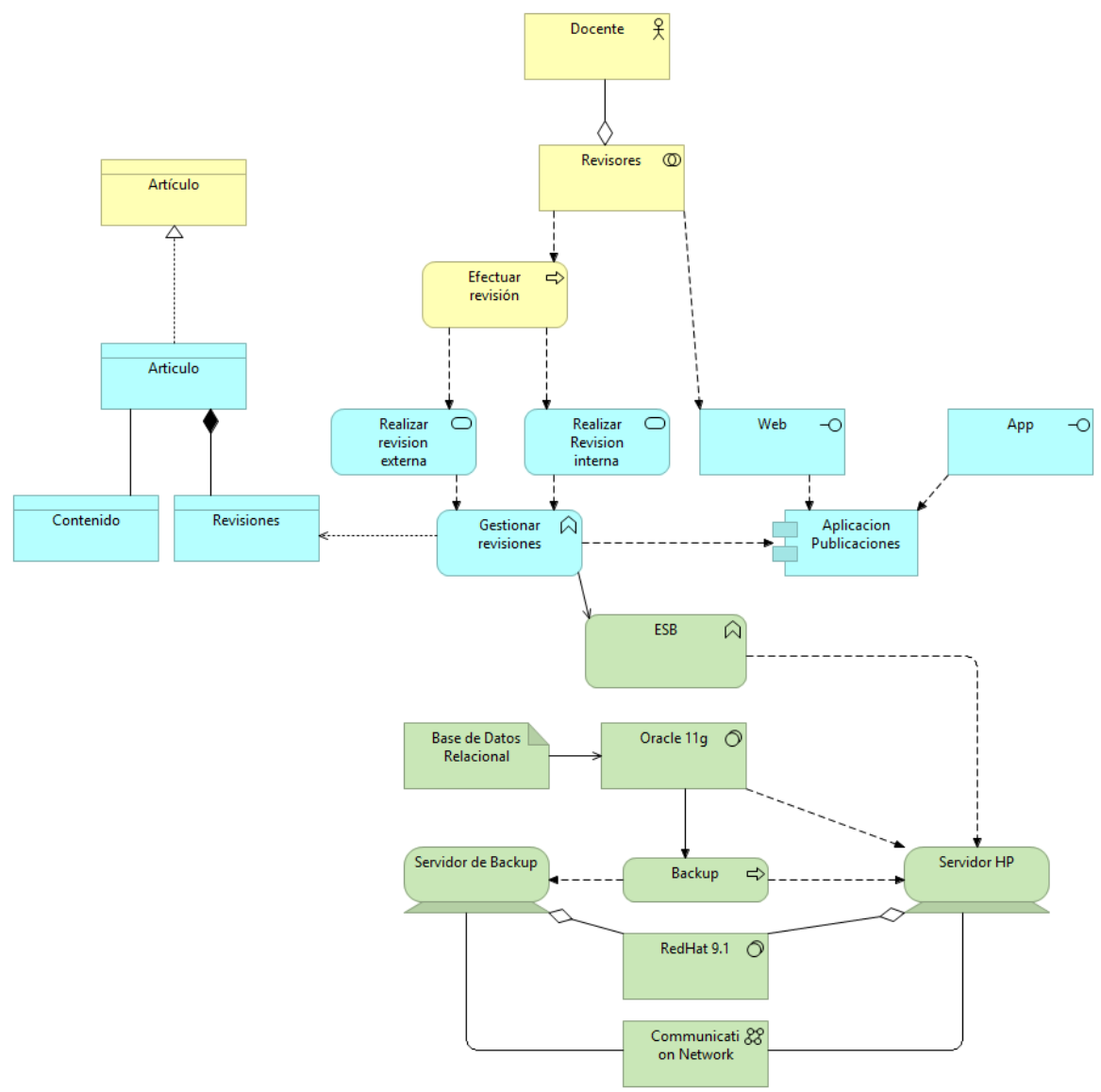

Figura 7. Modelamiento de la vista de aplicaciones y vista física 


\subsection{Fase E y F : ROADMAP}

La última fase consistió en definir la planificación de la arquitectura, esta se desarrolla a través de un Roadmap (ver figura 8) u hoja de ruta que presenta en el tiempo como se irá implementando la tecnología dentro de la organización, se elabora en base al análisis de la fase C y D.

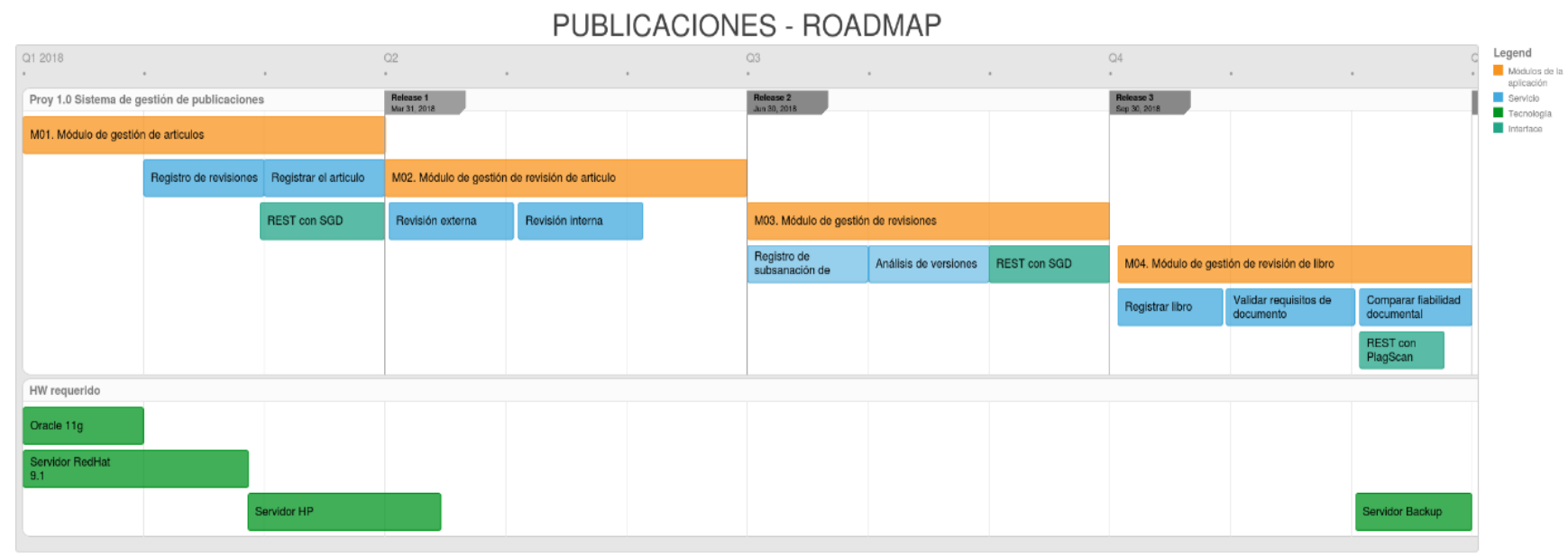

Figura 8. Roadmap propuesto de actividades para lograr los procesos ToBe 


\section{Conclusiones}

1. El análisis de visión de arquitectura permitió que la organización pueda identificar sus necesidades, propósitos de la organización y a partir de ella analizar los procesos, tecnología, aplicaciones e información requerida que permitirá lograr cumplir con el concerns de las publicaciones de investigación científica a mediano plazo.

2. Se elaboró un análisis de capacidades de la organización basado en los procesos que permitió un análisis desde una vista de bajo nivel cómo funciona la organización al interior de ella, a través de la identificación de procesos (Procesos ASIS), permitiendo de esta manera proponer mejoras y optimizar los procesos bajo un análisis no manual sino con una visión automatizada (Procesos TO BE).

3. Se logró alinear de manera integral los objetivos visiónales con la utilización de las tecnologías de información elaborando una vista integral que cubrió desde el proceso de negocio, en este caso las publicaciones, los sistemas de información propuestos, los datos e información de manera que logren posicionar a la institución a través de una visibilidad aceptable con el soporte de una infraestructura tecnológica

4. Finalmente, el desarrollar un modelo de arquitectura empresarial, utilizando el ADM de Togaf, ha permitido que se pueda optimizar los procesos, definir la tecnología requerida alineada a los objetivos organizacionales, adicionalmente permite visualizar en el tiempo como dicha tecnología se irá entregando e integrando a la organización.

\section{Referencias}

Arango, M. D, Londono, J. E \& Zapata, J. A. (2010). Arquitectura empresarial: una visión general. Rev. ing. univ. Medellín. vol.9, n.16, pp.101-111. 332

Ares, J. (2013). Intruduccion a TOGAF para el desarrollo de enterprise Architecture. Recuperado de: https://es.slideshare.net/netmindit/introduccin-a-togaf-para-el-desarrollo-de-enterprise-architecture

Jaramillo D, Cabrera A, Abad M, \&Torres A. (2016). Definición de un marco de referencia de ciberseguridad empresarial basado en ADM-TOGAF. (pp. 262-667). Ecuador. Conferencia Ibérica de Sistemas e Tecnologias de Informacion. Recuperado de:

https://www.researchgate.net/profile/Danilo_Jaramillo/publication/282329839_Definition_of_cybersecu rity_businness_framework_based_on_ADM-TOGAF/links/560c489b08aed543358d2c48/Definition-ofcybersecurity-businness-framework-based-on-ADM-TOGAF.pdf

Lankhorst et al, (2005). Entreprise Architecture at work, Modelling, communication, and analysis. Germany: Springer, (chapter 1).

Mejía, M. (2013). Arquitectura empresarial, El camino hacia un gobierno integrado recuperado de 
http://www.mintic.gov.co/gestionti/615/articles-5322_Revista_pdf.pdf

Niemi, E. \& Pekkola, S. (2016). Enterprise Architecture Benefit Realization: Review of the Models and a Case Study of a Public Organization recuperado de: http://dl.acm.org/citation.cfm?id=2980787

OpenGroup. (2011). Togaf 9.1. recuperado de The Open Group Architecture Framework, recuperado de: https://wikivisually.com/wiki/The_Open_Group_Architecture_Framework

Zachman, J. A. (1987). A framework for information systems architecture. Recuperado de https://www.zachman.com/images/ZI_PIcs/ibmsj2603e.pdf 